\title{
氮化铝覆铜板在空间热场下热学性能的模拟仿真及实验验证
}

\author{
何端鹏 ${ }^{1}$, 高 鸿 $^{1}$, 张静静 ${ }^{1}$, 吴 杰 $^{2}$, 刘泊天 ${ }^{1}$ ，王向轲 ${ }^{1}$
}

(1. 中国空间技术研究院 材料可靠性中心, 北京 100094; 2. 山东航天电子技术研究所, 烟台 264670)

摘 要: 氮化铝(AIN)陶瓷具有高导热、高电阻率、良好的尺寸稳定性以及优异的力学性能等特性, 被认为是新一代 高性能陶瓷基板和封装的首选材料。本研究探讨了高性能陶瓷在空间电子系统的应用潜力, 对 AlN 材料的基础性 能进行了分析, 重点分析了 AlN 陶瓷材料及其覆铜板的热传导性能, 从理论上分析了 AlN 材料及覆铜板的热特性, 并通过仿真模拟对理论值进行了分析验证，最后探讨了 AIN 陶瓷覆铜板在空间热循环模拟环境下的热传导性能。 结果表明 $\mathrm{AlN}$ 陶瓷的导热系数高达 $174.1 \mathrm{~W} \cdot \mathrm{m}^{-1} \cdot \mathrm{K}^{-1}$, 覆铜板比纯氮化铝陶瓷具有更高的热扩散系数, 而热特性的 仿真结果与理论计算一致。最后空间环境模拟试验表明, AlN 材料在温度循环环境下的热传导性能非常稳定。

关＼cjkstart键＼cjkstart词: 空间; 氮化铝; 覆铜板; 热导率; 理论计算; 仿真

中图分类号: TQ174 文献标识码: A

\section{Simulation and Experimental Verification of Thermal Property for Aluminum Nitrides and Copper Clad Laminates under Space Thermal Environment}

\author{
HE Duan-Peng ${ }^{1}$, GAO Hong ${ }^{1}$, ZHANG Jing-Jing ${ }^{1}$, WU Jie ${ }^{2}$, LIU Bo-Tian ${ }^{1}$, WANG Xiang-Ke ${ }^{1}$
}

(1. Material Reliability Center, China Academy of Space Technology, Beijing 100094, China; 2. Shandong Institute of Space Electronic Technology, Yantai 264670, China)

\begin{abstract}
Aluminum nitrides (AIN), which possess high thermal conductivity, high electrical resistivity, good dimensional stability and excellent mechanical property, have been considered the preferred materials as a new generation of high-performance ceramic substrate and packaging materials. In this paper, the application potential of ceramics in space electronic systems is discussed. And the basic capabilities of AlN were analyzed. Heat transfer property of AlN and its copper clad laminate were of selective and theoretical analysis, which were further verified by simulation. Finally, the thermal conductive performance of AIN in the simulated space thermal cycle environment was discussed. The results show that the thermal conductivity is up to $174.1 \mathrm{~W} \cdot \mathrm{m}^{-1} \cdot \mathrm{K}^{-1}$ and the thermal diffusivity of copper clad laminates is higher than that of pure aluminum nitrides. The simulation results of thermal characteristics are in agreement with the theoretical calculation. The final space environment simulation tests indicate that the thermal conductive capabilities of aluminum nitrides remain extremely stable.
\end{abstract}

Key words: aerospace; aluminum nitride; copper clad laminate; thermal conductivity; theoretical calculation; simulation

为响应我国航天器发展的新趋势，型号卫星的 电源控制器也朝着高频、高密度、高功率、高可靠 性、微型化、多功能化方向发展。然而, 系统的密
度及功率的增加则会凸显散热问题: 结构损坏、性 能恶化、甚至烧毁等。而器件的散热性能依赖于散 热结构的设计、散热材料(散热基板)的选用及封装

收稿日期：2018-11-29; 收到修改稿日期：2019-1-22

基金项目：中国空间技术研究院宇航物资保障事业部自主研发项目(ZY-WZB-2018-05)

Independent Research and Development of China Aerospace Components Engineering Center (ZY-WZB-2018-05)

作者简介：何端鹏 (1990-), 男, 硕士，工程师. E-mail: hedp09@163.com

通讯作者：高 鸿，高级工程师. E-mail: gaohong_cast@sina.com 
的工艺等。其中, 基板材料的选用是关键环节, 不仅 影响器件的成本, 而且影响器件的性能及可靠性。 电源控制器起到调控能量在太阳能电池阵、分流器、 电池充电调节器、电池放电调节器以及蓄电池之间 进行适当分配的作用, 从而使主母线上的电压稳定 在一个恒定的水平, 并为星上设备提供能量。

航天器的电源控制器为了节省空间, 不断提升 元器件的安装密度, 功率晶体管、功率二极管都采 用表面贴装(SMD)的方式组装, 而且功耗大于 $1 \mathrm{~W}$ 的所有表面贴装的功率晶体管、功率二极管都采用 高性能陶瓷板作为导热基板。选用高性能陶瓷板的 原因包括：1) 不能直接将 SMD 封装器件安装在机 壳上散热, 只能将其焊装在高导热载体上, 再将载 体安装在机壳上。2) 常用的印制板材料 FR-4 的线 膨胀系数较高 (一般大于 $10^{-5} / \mathrm{K}$ ), 与 SMD 器件材料 的线膨胀系数 (一般为 $(4 \sim 7) \times 10^{-6} / \mathrm{K}$ ) 不匹配, 若在 FR-4 材料的印制板上直接焊装器件, 有造成焊点开 裂或器件本体开裂的风险, 降低产品的可靠性。因 此设计电源控制器产品时, 使用导热性能优良、尺 寸稳定的陶瓷基板可解决与 SMD 封装器件的散热 以及线膨胀系数匹配性的问题。

传统的陶瓷基板材料有氧化铝 $\left(\mathrm{Al}_{2} \mathrm{O}_{3}\right)$ 陶瓷和氧 化铍 $(\mathrm{BeO})$ 陶瓷, 但是 $\mathrm{Al}_{2} \mathrm{O}_{3}$ 陶瓷基板导热率很低 $(\sim 30 \mathrm{~W} /(\mathrm{m} \cdot \mathrm{K}))$, 而且线膨胀系数与 $\mathrm{Si}$ 不相匹配。纯 度大于 $99 \%$ 、致密度 $99 \%$ 以上的 $\mathrm{BeO}$ 陶瓷, 其室温 下的导热率可达 $310 \mathrm{~W} /(\mathrm{m} \cdot \mathrm{K})$, 但是生产成本高且具 有毒性, 导致它的推广和应用受到限制 ${ }^{[1]}$ 。氮化铝 (AlN)陶瓷具有导热率高、热膨胀小、电阻率高、优 异的耐热震性及良好的力学性能等特性, 被认为是 新一代 “高性能”、“性能一体化” 的理想导热基板 材料 ${ }^{[2]}$ 。在国外, 19 世纪 70 年代首次制备合成了 AlN 粉体, 直到 20 世纪 50 年代, 才首次制得 AlN 陶瓷。 由于当时粉体制备方法及烧结技术的限制, 材料的 致密度较低, 机械强度也不高。但随着人们对非氧 化物陶瓷研究的重视, AlN 逐渐开始得到广泛的发 展。空间电源控制器可优选高性能 $\mathrm{AlN}$ 陶瓷导热基 板。导热基板由 AlN 基材板上下分别覆上等厚度的 铜层组成, 如图 1(a)红色虚线框所示, 氮化铝陶瓷 基板实物如图 1(b)所示。本研究探讨了高性能陶瓷 在空间电子系统的应用潜力, 选取氮化铝及其覆铜 板作为研究对象, 对 AlN 材料的基础性能进行了分 析, 重点分析了 AlN 陶瓷材料及其覆铜板的热传导 性能, 从理论上分析了 AlN 材料对体系热传导的影 响, 并通过仿真模拟对理论计算进行了验证, 最后 探讨了 AlN 陶瓷覆铜板在空间热交变环境中导热性 能的稳定性。

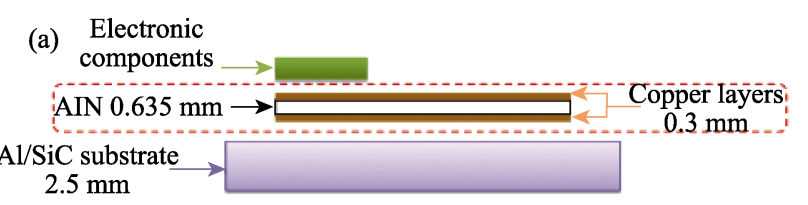

(b)

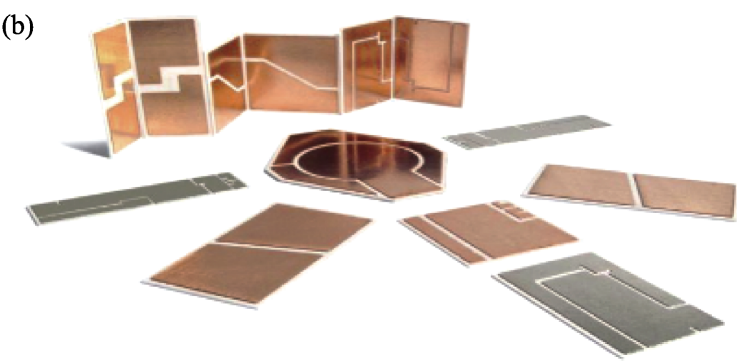

图 1 氮化铝陶瓷覆铜板(a)示意图及(b)实物图

Fig. 1 (a) Schematic diagram and (b) physical diagram of aluminum nitrides direct bonded copper

\section{1 实验方法}

\section{1 实验材料}

实验材料为韩国 $\mathrm{KIC}$ 公司生产的氮化铝(成分: $97 \% \mathrm{AlN}+3 \% \mathrm{Y}_{2} \mathrm{O}_{3}$ )及其覆铜板。

\section{2 性能测试}

用场发射扫描电镜(MEILIN 6169, Zeiss)对氮化 铝陶瓷进行微观形貌观察。采用 $X$ 射线衍射仪 (X-ray diffraction, Rigaku)对试样进行相结构分析, 扫描范围 $2 \theta=10^{\circ} \sim 80^{\circ}$, 扫描速率 $5\left(^{\circ}\right) / \mathrm{min}$ 。用 MTS6104 型电子 万能试验机测定样品的弯曲强度及弹性模量。基于 阿基米德原理采用排水法测定氮化铝陶瓷的密度 $(\rho)$, 用差示扫描量热仪(DSC 200 F3 NETZSCH)测 试氮化铝陶瓷的比热容 $\left(C_{\mathrm{p}}\right)$, 采用激光闪射仪 (LFA $467, \mathrm{NETZSCH})$ 测试氮化铝陶瓷的热扩散系数 $(\beta)$, 并通过如下公式(1)计算得到导热系数。

\section{3 仿真模拟分析}

$$
\lambda=\rho \times C_{\mathrm{p}} \times \beta
$$

温度场仿真模拟分析采用有限元分析软件。为 了更好地分析电源控制器中使用到陶瓷覆铜板的温 度场, 避免不必要因素的影响, 减少计算量, 对体 系进行了以下简化与假设: (1)电源控制器由于工作 发热传输的热量以温度形式均匀地传导; (2)假设陶 瓷材料的热导率是完美的各向同性。

\section{4 空间环境模拟试验}

主要模拟了空间温度循环环境。试验过程为: 将温度传感器贴于试件表面, 并对试件进行了预烘 去潮处理, 温度 $70{ }^{\circ} \mathrm{C}, 1 \mathrm{~h}$ 。设置试验条件: (1)温度 范围是 $-55 \sim+100{ }^{\circ} \mathrm{C}$; (2)循环次数 500 次; (3) 热循环 温度梯度不超过 $10{ }^{\circ} \mathrm{C} / \mathrm{min}$; (4)高低温极限温度停留 时间 15 min。 


\section{2 结果与讨论}

\section{1 氮化铝陶瓷性能}

采用 $\mathrm{X}$ 射线对试样进行晶体结构分析, 本实验 对二组试样分别进行了检测。图 2 为检测结果, 两 组试样分别标识为 XRD-1 及 XRD-2, 两组试样的图 谱基本一致, 说明材料均匀性较好, 各组次一致。通 过 $X$ 射线标准图谱比对可看出，探测到的特征峰与 AlN 标准卡片(JCPDS NO 25-1133)特征峰一致。除 此之外, 从 XRD 衍射峰中也能识别出 YAG 相 $\left(3 \mathrm{Y}_{2} \mathrm{O}_{3} / 5 \mathrm{Al}_{2} \mathrm{O}_{3}\right)$ 的存在 ${ }^{[3-5]}$, 与制备工艺中助剂的添 加吻合。值得注意的是, 为了提升 AIN 陶瓷的致密 度, 降低烧结温度, 一般通过加入烧结助剂, 促进 形成晶界相, 可有效降低烧结温度。

图 3(a)为 AlN 陶瓷断面扫描电镜照片, 从照片 可以看出, 氮化铝形貌呈现出完善的多面体颗粒状 晶粒, 晶粒之间面面紧密接触, 致密程度较高, 晶 界棱边清晰。研究证明, $\mathrm{AlN}$ 是比较稳定的, 但 $\mathrm{AlN}$ 与氧具有较强的化学亲和性, 因此 AlN 容易被氧化, 氧化速率取决于 AlN 本体材料的活性及其表面状态, AlN 中氧的存在会导致本体材料性能的退化 ${ }^{[6-7]}$ 。见 图 3(b)示意图, 从结构决定性能的角度分析, 示意 图揭示了晶界状态对导热系数的影响机理: 制备工 艺中不加助剂, 晶界形成大量包含残余氧的固溶体 及尖晶石层, 导热系数最小 $\left(\lambda=80 \sim 100 \mathrm{~W} \cdot \mathrm{m}^{-1} \cdot \mathrm{K}^{-1}\right)$; 制备工艺中添加助剂, 晶界只形成包含残余氧的石 榴石窄带，导热系数较大 $\left(\lambda=150 \sim 200 \mathrm{~W} \cdot \mathrm{m}^{-1} \cdot \mathrm{K}^{-1}\right)$; 制备工艺中添加助剂以及还原气氛, 去除晶界残余 氧, 导热系数最大 $\left(\lambda=240 \sim 270 \mathrm{~W} \cdot \mathrm{m}^{-1} \cdot \mathrm{K}^{-1}\right)^{[8-9]}$ 。从氮 化铝的晶界状态可看出属于后两种类型的形貌, 氮 化铝具有较高的导热系数。

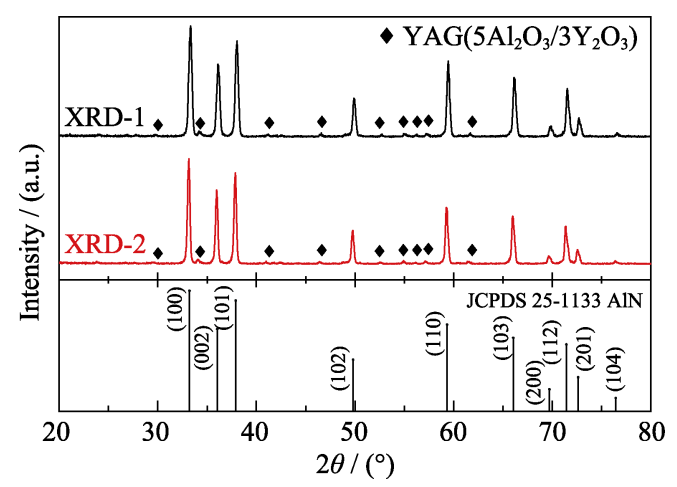

图 2 AlN 陶瓷的 XRD 图谱

Fig. 2 XRD patterns of AlN ceramics

氮化铝陶瓷基材的力学性能检测结果显示，室 温弯曲强度及弹性模量的平均值和离散系数分别为: $446 \mathrm{MPa} / 4.3 \% 、 317 \mathrm{GPa} / 1.1 \%$, 说明氮化铝材料的 力学性能优异, 而且检测数据离散度很小, 证实氮 化铝陶瓷的均匀性较好。

\section{2 导热性能}

由 AlN 陶瓷体积密度 $\rho$ (检测结果平均值为 $3.296 \mathrm{~g} / \mathrm{cm}^{3}$, 离散系数为 $0.015 \%$ )、比热容 $C_{\mathrm{p}}$ 及热 扩散系数 $\beta$ 计算得到材料的导热系数, 导热系数随 温度的变化曲线如图 4, AlN 陶瓷在-55 100 ${ }^{\circ} \mathrm{C}$ 下的 导热系数在低温和高温时具有较大的差异, 呈现出 温度升高, 其导热系数逐渐减小的规律。常温下, AlN 陶瓷的导热系数为 $174.1 \mathrm{~W} \cdot \mathrm{m}^{-1} \cdot \mathrm{K}^{-1}$, 检测结果 证实了上述扫描电镜微观形貌观察中的结构决定性 能的分析。

图 5(a)为氮化铝陶瓷及其覆铜板的实物照片, 从外观上观察基板表面无裂纹、污点、刮伤等表面 缺陷。覆铜板为氮化铝陶瓷双面覆铜而成, 铜层与 氮化铝陶瓷结合牢固, 不存在裂缝。进一步采用激 光闪射仪检测得到了覆铜板热扩散系数。同时将覆

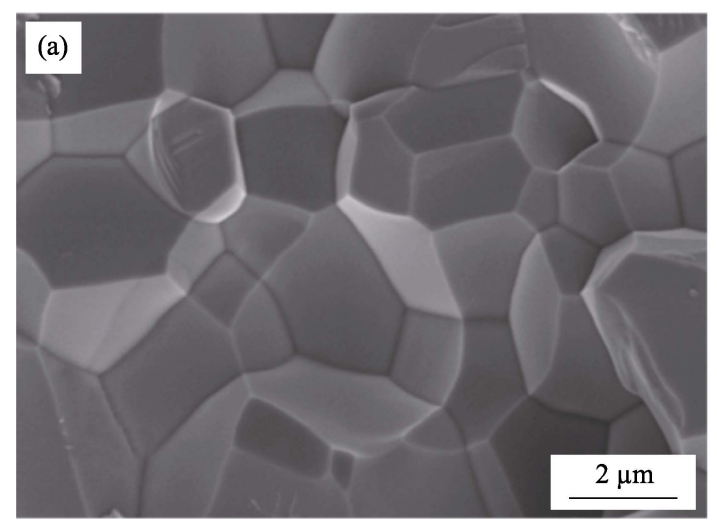

(b)
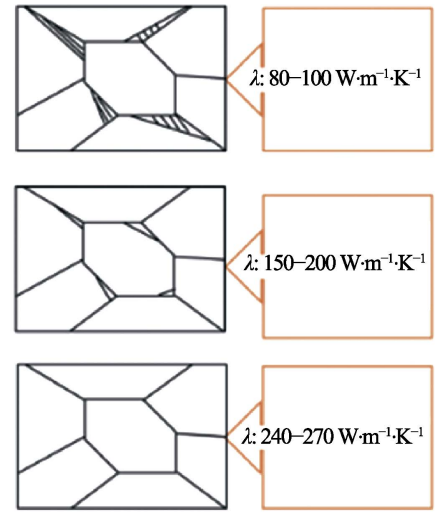

图 3 (a)AIN 的微观晶粒形貌及(b)晶界对导热系数的影响机理示意图

Fig. 3 (a) Grain morphology of AlN and (b) schematic diagram of the mechanism of the effect of grain boundary on thermal conductivity 


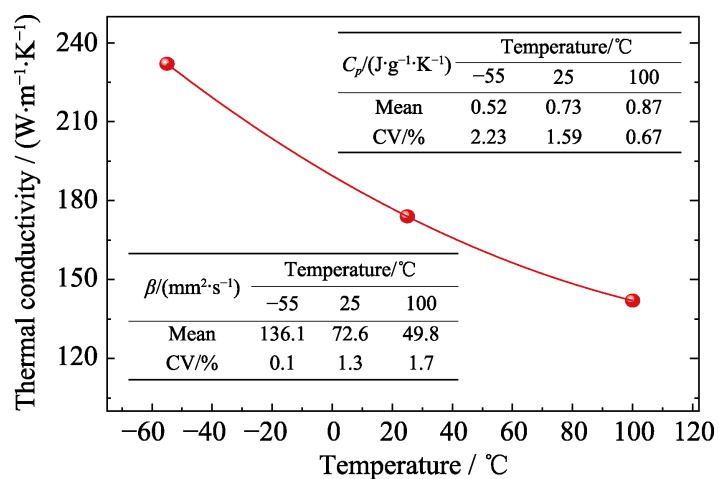

图 4 不同温度下 AlN 陶瓷的导热系数, 插图为比热容及热 扩散的检测数据

Fig. 4 Thermal conductivities of AIN ceramics at different temperatures with insets showing the measured data of specific heat capacity and thermal diffusion

(a)
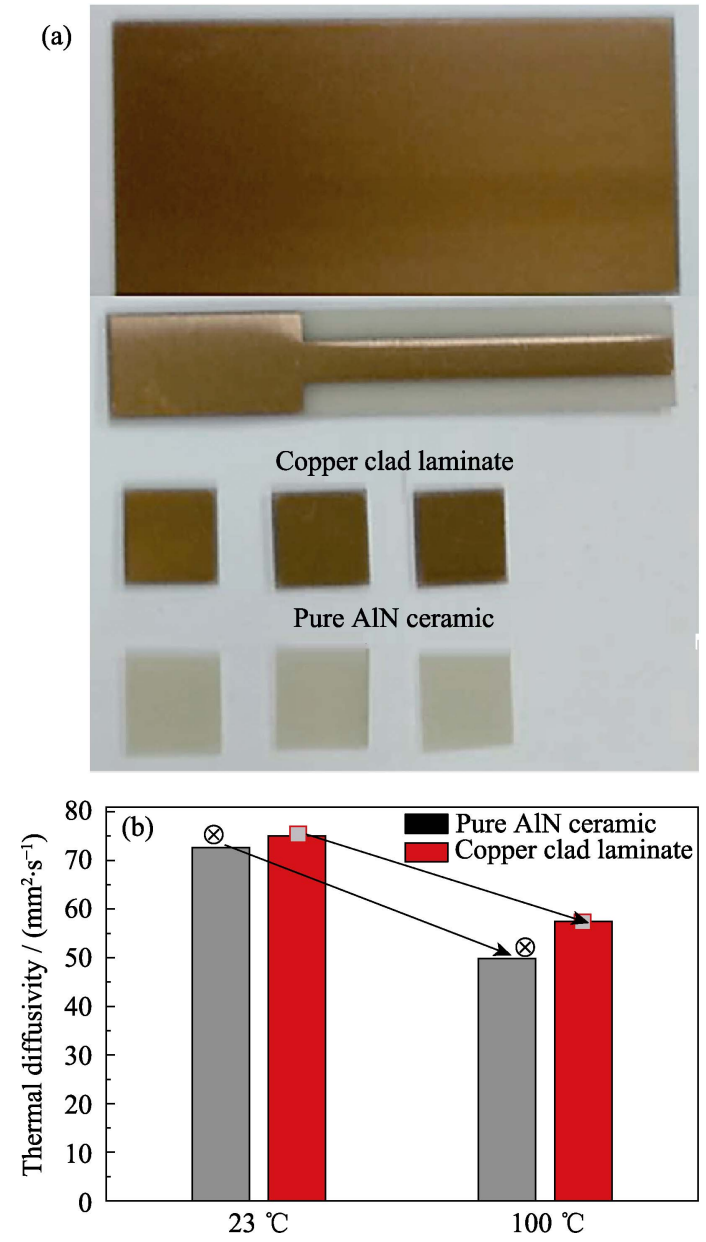

图 5 氮化铝陶瓷及其覆铜板的(a)实物照片和(b)热扩散系数

Fig. 5 (a) Photographs and (b) thermal diffusion coefficient of AlN ceramics and copper clad laminate

铜板与纯氮化铝的热扩散系数进行对比分析，见 图 5(b), 说明覆铜板与纯氮化铝的热扩散系数都随 着温度的升高而降低。但在检测的温度范围内, 覆 铜板相比氮化铝具有更优的热扩散性能，这是由于 铜在室温及高温具有比氮化铝更高的热扩散系数
$\left(\mathrm{Cu}: \sim 110 \mathrm{~mm}^{2} / \mathrm{s}, \mathrm{AlN}: 50 \sim 80 \mathrm{~mm}^{2} / \mathrm{s}\right)^{[10]}$, 因此, 氮化 铝经过表面覆铜，比氮化铝陶瓷具有更高的热扩散 系数。

\section{3 氮化铝陶瓷及覆铜板热特性理论分析}

图 6 为陶瓷覆铜板安装于机箱的三维模型, 其 中, 黑色为电子元器件, 白色为氮化铝覆铜板, 黄 色为铝基碳化硅衬底, 蓝色为机箱外壳。该体系的 传热路径为: 热量通过元器件引线导热及元器件与 印制电路板之间的接触传热传到氮化铝陶瓷覆铜板, 通过覆铜板再传到衬底, 最终传到机箱。陶瓷覆铜 板安装于机箱中不同位置, 考虑最恶劣工况, 各陶 瓷覆铜板热耗 $Q_{\mathrm{k}}$ 分别为: $16.3 、 28.7 \mathrm{~W}$ 。陶瓷板 1 的 尺寸为: $37.7 \mathrm{~mm} \times 27.2 \mathrm{~mm}$, 面积为 $A_{1}=1025.44 \mathrm{~mm}^{2}$, AlN 厚度及铜层厚度分别为 $X_{\mathrm{AlN}}=0.635 \mathrm{~mm} 、 X_{\mathrm{Cu}}=$ $0.3 \mathrm{~mm}+0.3 \mathrm{~mm}=0.6 \mathrm{~mm}$; 陶瓷板 2 的尺寸为： $54.4 \mathrm{~mm} \times 27.2 \mathrm{~mm}$, 面积为 $A_{2}=1479.68 \mathrm{~mm}^{2}, \mathrm{AlN}$ 厚 度及铜层厚度分别为 $X_{\mathrm{AlN}}=0.635 \mathrm{~mm} 、 X_{\mathrm{Cu}}=0.3 \mathrm{~mm}+$ $0.3 \mathrm{~mm}=0.6 \mathrm{~mm}$ 。祄底厚度为 $2.5 \mathrm{~mm}$, 壳体厚度 $5 \mathrm{~mm}$ 。

根据航天器热设计公式(2)、(3) ${ }^{[11]}$ :

$$
\begin{gathered}
\Delta T=Q_{k} \cdot R_{k} \\
R_{k}=\frac{\Delta X}{A \cdot \kappa}
\end{gathered}
$$

式中: $Q_{k}$ 一热耗(热流), $\mathrm{W} ; R_{k}$ 一导热热阻, ${ }^{\circ} \mathrm{C} / \mathrm{W}$; $\Delta T$ 一在传热方向的温度差, ${ }^{\circ} \mathrm{C} ; \Delta X$ 一导热路径长 度, $\mathrm{m}$ 。

计算陶瓷板 1 的热阻 $R_{1}$ 为:

$$
\begin{gathered}
R_{1}=2 R_{\mathrm{Cu}}+R_{\mathrm{AIN}}=2 \times \frac{0.3 \times 10^{-3}}{1025.44 \times 10^{-6} \times 395}+ \\
\frac{0.635 \times 10^{-3}}{1025.44 \times 10^{-6} \times 170}=0.0051\left({ }^{\circ} \mathrm{C} / \mathrm{W}\right)
\end{gathered}
$$

计算陶瓷板 2 的热阻 $R_{2}$ 为:

$$
\begin{gathered}
R_{2}=2 R_{\mathrm{Cu}}+R_{\mathrm{AIN}}=2 \times \frac{0.3 \times 10^{-3}}{1479.68 \times 10^{-6} \times 395}+ \\
\frac{0.635 \times 10^{-3}}{1479.68 \times 10^{-6} \times 170}=0.0035\left({ }^{\circ} \mathrm{C} / \mathrm{W}\right)
\end{gathered}
$$

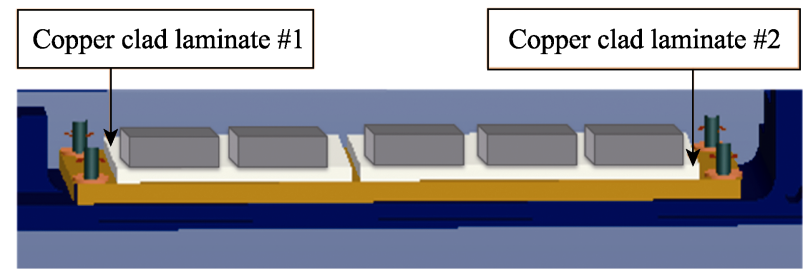

图 6 陶瓷覆铜板三维模型

Fig. 6 3D model of ceramic copper clad laminate 
因此，电源控制器工作时，以陶瓷覆铜板 1 作 为传热通道, 工作器件与祄底的最大温差为:

$$
\Delta T_{1}=Q_{1} \cdot R_{1}=28.7 \times 0.0051=0.15{ }^{\circ} \mathrm{C}
$$

以陶瓷覆铜板 2 作为传热通道, 工作器件与祄 底的最大温差为:

$$
\Delta T_{2}=Q_{1} \cdot R_{2}=28.7 \times 0.0035=0.10{ }^{\circ} \mathrm{C}
$$

通过上述理论计算发现, 考虑在最极端最恶劣 工况下, 由于氮化铝陶瓷出色的导热性能, 工作器 件与祄底最大的温差仅为 $0.10 \sim 0.15{ }^{\circ} \mathrm{C}$, 说明器件 工作产生的热量能够快速通过该氮化铝覆铜板传导 至祄底。

针对模块的等效热阻网络如图 7, 系统的总热 阻为:

$$
\begin{gathered}
R=R_{\mathrm{C} 1}+R_{\mathrm{Cu}-\mathrm{AlN}}+R_{\mathrm{C} 2}+R_{\mathrm{SiC} / \mathrm{Al}}+ \\
R_{\mathrm{C} 3}+R_{\text {solder, coating }}+R_{\text {outer shell }}
\end{gathered}
$$

由上述分析可知, 系统的总热阻取决于陶瓷覆 铜板 $\left(R_{\mathrm{Cu}-\mathrm{AIN}}\right)$ 、衬底 $\left(R_{\mathrm{SiC} / \mathrm{Al}}\right)$ 、焊料、镀层 $\left(R_{\text {solder, coating }}\right)$ 和外壳 $\left(R_{\text {outer shell }}\right)$ 的热阻以及各部件 间的接触热阻 $\left(R_{\mathrm{c}}\right)$ 。为简化进一步的理论分析, 忽略 各部件间的接触热阻 $R_{\mathrm{c}}$, 根据公式(2), 计算出各部 件的热物理参数见表 1 。

因此，系统工作时，器件与外壳之间最大的温 差为:

$$
\begin{gathered}
\Delta T_{\max }=Q_{\max } \cdot R=Q_{\max } \cdot\left(R_{C 1}+R_{\mathrm{Cu}-\mathrm{AlN}}+R_{\mathrm{C} 2}+\right. \\
\left.R_{\mathrm{SiC} / \mathrm{Al}}+R_{\mathrm{C} 3}+R_{\text {solder, coating }}+R_{\text {outer shell }}\right) \approx 28.7 \times \\
(0.0051+0.0136+0.0033+0.1394)=4.63{ }^{\circ} \mathrm{C}
\end{gathered}
$$

当外壳温度(环境温度)为 $T_{0}$ 时, 通过上述理论 分析可知, 内部器件温度比外壳温度高 $4.63{ }^{\circ} \mathrm{C}$ 。

\section{4 热仿真分析}

基于 2.3 中的理论分析，进一步通过仿真模拟 分析系统的热特性。设置机箱外壳(环境温度)温度 为 $60{ }^{\circ} \mathrm{C}$, 仿真过程忽略螺钉、引线等的影响。仿真 运算获得控制器工作时的热场分布如图 8(a)所示, 从结果看出, 在器件工作热耗为 $28.7 \mathrm{~W}$ 的条件下, 当机箱外壳温度为 $60{ }^{\circ} \mathrm{C}$ 时, 器件的温度为 60.33

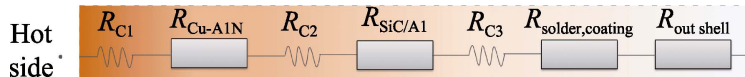

Cold side

图 7 模块的等效热阻网络

Fig. 7 Equivalent thermal resistance network of modules

表 1 各部件的热物理参数

Table 1 Thermophysical parameters of components

\begin{tabular}{cccc}
\hline $\begin{array}{c}\text { Materials } \\
\begin{array}{c}\text { Module compo- } \\
\text { nents) }\end{array}\end{array}$ & $\begin{array}{c}\text { Thermal } \\
\text { conductivity } \\
/\left(\mathrm{W} \cdot \mathrm{m}^{-1} \cdot \mathrm{K}^{-1}\right)\end{array}$ & $\begin{array}{c}\text { Thickness } \\
/ \mathrm{mm}\end{array}$ & $\begin{array}{c}\text { Thermal } \\
\text { resistance } \\
/\left({ }^{\circ} \mathrm{C} \cdot \mathrm{W}^{-1}\right)\end{array}$ \\
\hline $\begin{array}{c}\text { Aluminum } \\
\text { nitrides direct } \\
\text { bonded copper }\end{array}$ & 170 & 1.235 & 0.0051 \\
$\begin{array}{c}\text { Supporting base } \\
\text { (SiC/Al) }\end{array}$ & 180 & 2.5 & 0.0136 \\
$\quad \begin{array}{l}\text { Solder } \\
\text { Shell (alumina) }\end{array}$ & 60 & 0.2 & 0.0033 \\
\hline
\end{tabular}

$65.27{ }^{\circ} \mathrm{C}$, 比外壳温度高 $5{ }^{\circ} \mathrm{C}$ 左右, 与理论计算结果 较为吻合。

氮化铝陶瓷及覆铜板经历 500 次 “ $-55{ }^{\circ} \mathrm{C} \rightarrow$ $+100{ }^{\circ} \mathrm{C} \rightarrow-55{ }^{\circ} \mathrm{C}$ ” 空间温度循环模拟试验，根据氮 化铝陶瓷热循环试验后材料属性的变化，同时假设 其他各部件材料的物性参数稳定, 不随温度变化而 变化，进一步仿真模拟分析了系统在经历 500 次热 循环后的热场分布。同样设置模型环境温度为 $60{ }^{\circ} \mathrm{C}$, 由图 8(b) 可看出, 器件的温度分布在 $60.32 \sim 65.30{ }^{\circ} \mathrm{C}$ 之间, 各处温度分布比较均匀, 比外壳温度依然只 高 $5{ }^{\circ} \mathrm{C}$ 左右, 说明经历热循环 500 次后，系统依然 保持与热循环前近似的温升，热传导性能稳定。

\section{5 空间温度循环环境耐受性验证}

材料性能的变化除了与材料的原始组织的变异 和测试误差有一定关系外, 也与可能产生的环境效 应有关。因此, 本研究进一步探讨了温度循环环境 对氮化铝材料性能的影响。对经历 500 次温度循环 试验后的 AlN 陶瓷材料的热传导性能进行了分析。 图 9 显示温度循环前后 AlN 材料的热扩散系数及导 热系数基本没有大的变化, 说明 AlN 材料在空间温度 循环环境下热传导性能非常稳定，几乎不受影响。
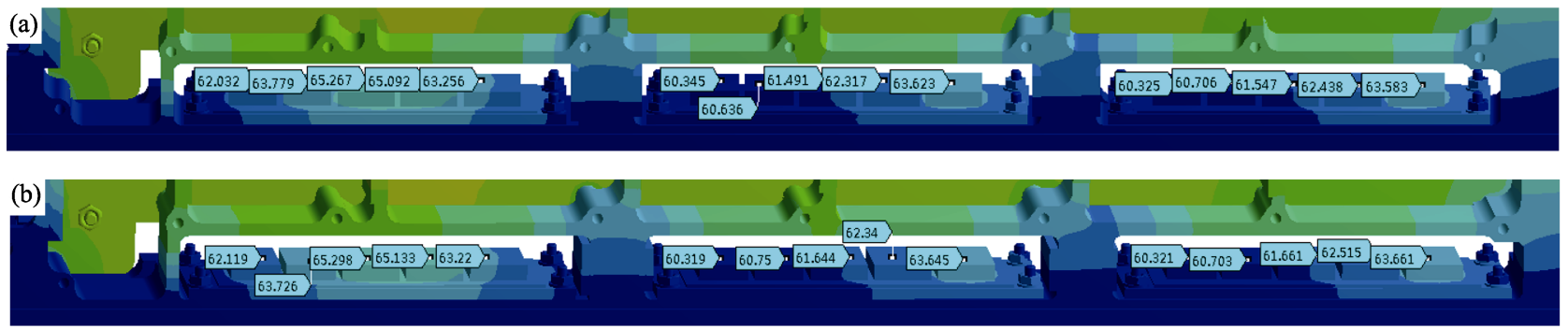

图 8 控制器热场分布仿真图

Fig. 8 Simulation diagrams of temperature distribution under (a) normal conditions and (b) after 500 thermal cycle tests 


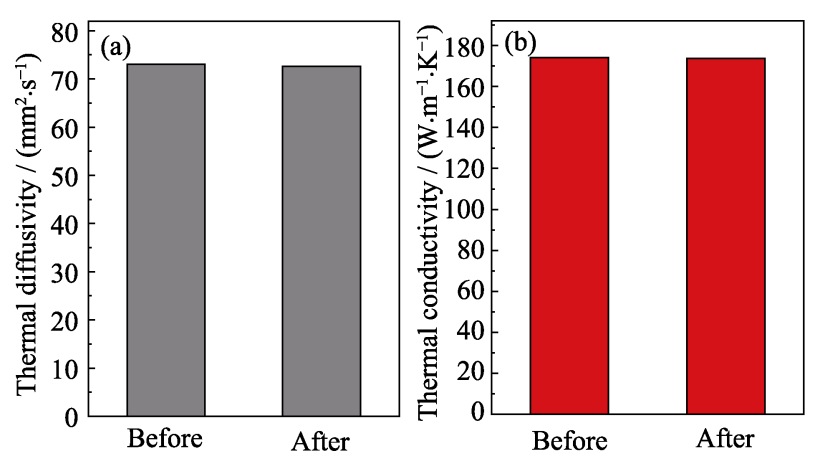

图 9 空间温度循环模拟试验对 (a)氮化铝陶瓷材料热扩散 和(b)热传导性能的影响

Fig. 9 Effect of space temperature cycle simulation test on (a) thermal diffusivity and (b) conductivity of aluminum nitrides
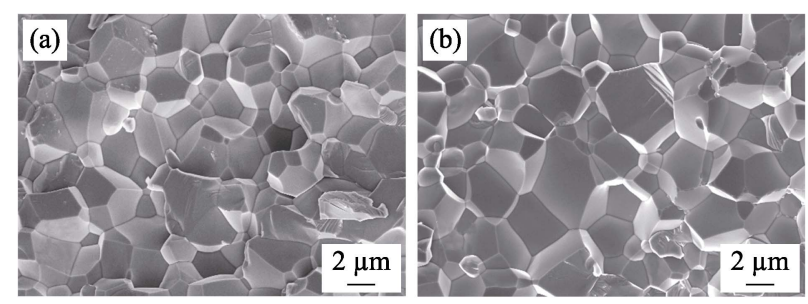

图 10 温度循环试验(a)前(b)后 AlN 陶瓷材料的扫描电镜 照片

Fig. 10 Scanning electron microscope photographs of AlN ceramics (a) before and (b) after temperature cycle test

同样从结构性能关系的角度探讨了 AIN 陶瓷材 料在温度循环试验前后的微观组织结构的变化。图 $10(a \sim b)$ 分别为温度循环试验前后的 A1N 陶瓷材料 的显微组织照片, 呈现出完善的多面体颗粒状晶粒, 致密程度较高, 晶界棱边清晰, 并且晶粒大小接近, 晶界状态基本一致, 说明温度循环试验前后 AlN 陶 瓷材料的微观组织无明显变化。根据前文分析, 可 从微观组织结构上说明 $\mathrm{AlN}$ 材料在温度循环环境下 热扩散系数及导热系数保持稳定的缘由。

\section{3 结论}

1)探讨了高性能氮化铝陶瓷在空间电子系统的 应用潜力。通过 XRD 分析、扫描电镜观察及导热 性能检测表明, 材料主成分为 AIN, 有 YAG 相的存
在, 陶瓷致密度高, 晶粒晶界棱边清晰, 电镜观察 到的晶界状态可推测材料具有较优异的导热性能, 实测导热系数高达 $174.1 \mathrm{~W} \cdot \mathrm{m}^{-1} \cdot \mathrm{K}^{-1}$, 而且氮化铝 覆铜板比纯氮化铝陶瓷具有更高的热扩散系数。

2) 氮化铝陶瓷覆铜板应用于器件时的热特性 仿真结果与理论计算吻合。

3) 空间环境模拟试验表明, AlN 材料在温度循 环环境下热传导性能保持稳定。

\section{参考文献:}

[1] CHENG HAO, CHEN MING-XIANG, HAO ZI-LIANG, et al. Progress of technologies and applications of ceramic substrate for the packaging of power electronics. Electronic Components and Materials, 2016, 35(1): 7-11.

[2] HE DUAN-PENG, GAO HONG, WANG XIANG-KE, et al. High thermal conductive and electric isolative aluminum nitrides and their application for space electronics. Aerospace Parts Engineering, 2017, 4(3): 186-192.

[3] LIU YE, QIN MING-LI, ZHANG LIN, et al. Solution combustion synthesis of $\mathrm{Ni}-\mathrm{Y}_{2} \mathrm{O}_{3}$ nanocomposite powder. Transactions of Nonferrous Metals Society of China, 2015, 25(1): 129-136.

[4] KUME S, YASUOKA M, LEE S K, et al. Dielectric and thermal properties of AIN ceramics. J. Eur. Ceram. Soc., 2007, 27(8/9): 2967-2971.

[5] HUANG LIN-YUN, LI CHEN-HUI, KE WEN-MING, et al. Effect of rare earth oxides on electrical properties of spark plasma sintered AlN ceramics. Journal of Inorganic Materials, 2015, 30(3): 267-271.

[6] HUANG M R S, ERNI R, LIU C P. Influence of surface oxidation on the valence electron energy-loss spectrum of wurtzite aluminum nitride. Appl. Phys. Lett., 2013, 102(6): 061902.

[7] JARRIGE J, LECOMPTE J P, MULLOT J, et al. Effect of oxygen on the thermal conductivity of aluminium nitride ceramics. J. Eur. Ceram. Soc., 1997, 17: 1891-1895.

[8] FUMIO MIYASHIRO, NOBUO IWASE, AKIHIKO TSUGE, et al. High thermal conductivity aluminum nitride ceramic substrates and packages. IEEE Transactions on Components, Hybrids, and Manufacturing Technology, 1990, 13(2): 313-319.

[9] XUE JIAN-FENG, LI JUN, ZHOU GUO-HONG, et al. Fabrication of aluminum nitride by electrophoretic deposition. Journal of Inorganic Materials, 2009, 24(6): 1151-1154.

[10] 刘光启, 马连湘, 刘杰. 化学化工物性数据手册, 无机卷, 2002.

[11] Thermal Design Handbook for Reliability of Electronic Equipment: GJB 27-92. 